\title{
Highly Selective Breath Acetone Analyzer Using ZnO Quantum Dots based Sensor with a Miniaturized Gas Chromatographic Column
}

\author{
Hwaebong Jung ${ }^{1}$, Wonhee Cho², Ran Yoo ${ }^{1}$, Hyun-Sook Lee ${ }^{1}$, Yong-Sahm Choe ${ }^{3}$, \\ Justin Y. Jeon ${ }^{2, *}$, Wooyoung Lee ${ }^{1, *}$ \\ ${ }^{1}$ Department of Materials Science and Engineering, Yonsei University, 50 Yonsei-ro, Seodaemun-gu, \\ Seoul, 03722, Republic of Korea \\ 2 Department of Sport Industry Studies, Yonsei University, 50 Yonsei-ro, Seodaemun-gu, Seoul, \\ 03722, Republic of Korea \\ 3 Isenlab Inc., Halla Sigma Valley, 545 Dunchon-daero, Jungwon-gu, Seongnam-si, Gyeonggi-do, \\ 13215, Republic of Korea, \\ *E-mail: wooyoung@yonsei.ac.kr(W.Lee), jjeon@yonsei.ac.kr (J.Y. Jeon)
}

\begin{abstract}
We report the development of a breath acetone analyzer using a sensor integrated with ZnO quantum dots (QDs) and a miniaturized gas chromatography (GC) column to separate acetone from human breath. The average size of the ZnO QDs, synthesized by a wet chemical method, is approximately 6 $\mathrm{nm}$. The analyzer can detect acetone concentration as low as $0.1 \mathrm{ppm}$. The response of the ZnO QDs increases with increasing acetone gas concentration and is strongly correlated with the concentration $\left(R^{2}=0.9915\right)$. Additionally, the miniaturized $\mathrm{GC}$ column can effectively separate acetone gas from the breath. The acetone content in the breath of volunteers (who were on a ketogenic diet for 3 days and a normal diet) was monitored using the breath acetone analyzer.
\end{abstract}

Key words: Breath acetone, $\mathrm{ZnO}$ quantum dot, miniaturized gas chromatography column, real-time detection, mini sampling volume.

\section{Introduction}

Breath analysis using gas chromatography (GC) to identify more than 200 compounds in the human breath came into practice in the 1970s [1]. Endogenous compounds such as inorganic gases (NO, CO) and VOCs (acetone, ethanol, ammonia, ethane and pentane) in the human breath serve as a biomarker for several diseases [2]. Breath acetone is related to diabetes [3], fasting [4], fat metabolism [5], and other numerous diseases [6].

The breath acetone concentration is measured by gas chromatography-mass spectrometry (GC-MS), selected ion flow tube mass spectrometry (SIFT-MS), ion mobility spectrometry (IMS), and proton transfer reaction mass spectrometry (PTR-MS), which are commonly used in the laboratory. With increasing interest in smart healthcare, portable personal healthcare devices are in high demand. The conventional tools for breath analysis are disadvantageous in terms of cost, portability, and complexity.

\begin{abstract}
Materials and Methods
Breath Acetone Analyzer: The breath acetone analyzer comprised a sampling loop, a packed column, three solenoid valves, a mini-sized pump, and a sensor based on $\mathrm{ZnO}$ QDs. Acetone is separated from the exhaled breath by the packed column within 100 seconds. The operation temperature of the column is maintained at $30{ }^{\circ} \mathrm{C}$. The separated acetone gas is detected by the $\mathrm{ZnO}$ QD-based sensor.
\end{abstract}

Acetone Gas Testing: The ZnO QD-based sensors were tested at various temperature $\left(394,417,430\right.$, and $\left.446{ }^{\circ} \mathrm{C}\right)$ optimize the operation temperature of the breath acetone analyzer system within the boundary. The breath acetone analyzer was used to detect air balanced acetone gas. The air balanced acetone gas was used to confirm the detection range of $\mathrm{ZnO}$ QD-based sensor across the wide range of acetone concentration (0.1-50 ppm) at optimized operation temperature.

Human Breath Analysis: Three healthy subjects (3 male volunteers) were tested. One volunteer was on the ketogenic diet (less than $4 \%$ of daily carbohydrate intake) for 3 days, and 
two volunteers were on a normal diet. After the ketogenic diet, the breath acetone was measured using the breath acetone analyzer.

\section{Results and Discussion}
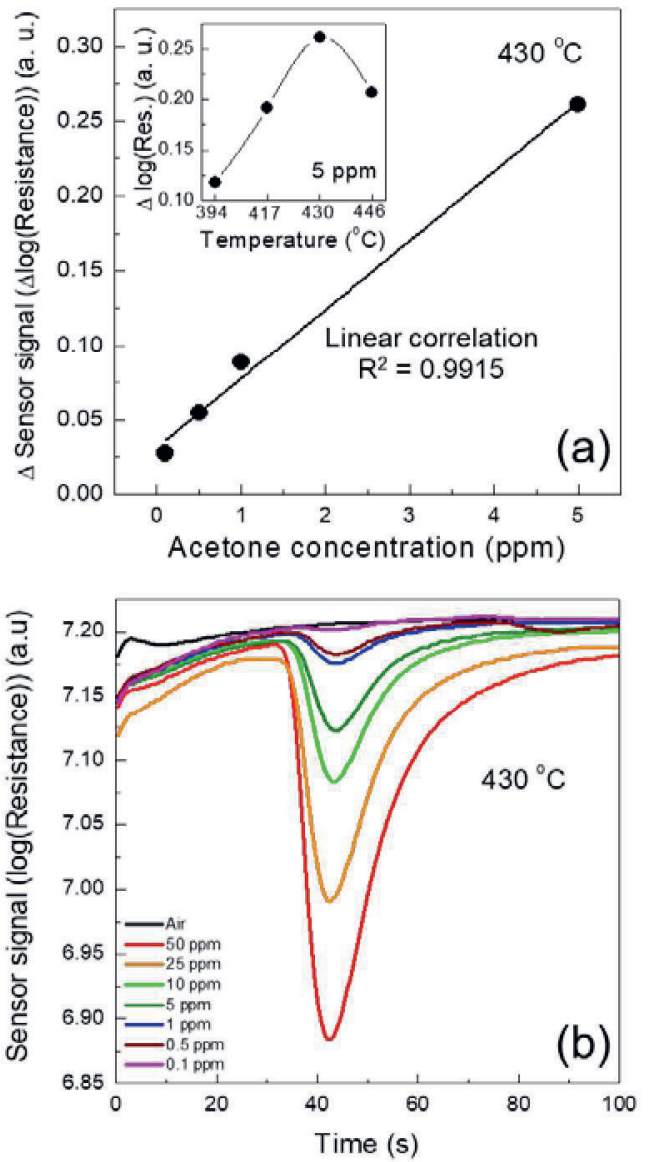

Fig. 1. (a) Responses with various acetone concentrations $(0.1-5 \mathrm{ppm})$ at $430{ }^{\circ} \mathrm{C}$ and response with $5 \mathrm{ppm}$ acetone at $394-446{ }^{\circ} \mathrm{C}$ (inset). (b) Output signal (log(Resistance)) change for various acetone concentrations (0-50 ppm), at $430{ }^{\circ} \mathrm{C}$.

Figure 1(a) shows the air balanced acetone gas responses $(\Delta(\log ($ Resistance $)))$ for various acetone gas concentration at $430^{\circ} \mathrm{C}$. Clearly, the response increased with increasing acetone gas concentration. The figure also shows the strong correlation $\left(R^{2}=0.9915\right)$ between the response and the acetone concentration. The inset image shows the air balanced acetone gas sensing properties at various temperatures. The response of the sensor increased with the operating temperature under $430{ }^{\circ} \mathrm{C}$ but decreased above $430{ }^{\circ} \mathrm{C}$. The optimal operating temperature of the $\mathrm{ZnO} \mathrm{QD}$ sensors was thus determined to be $430{ }^{\circ} \mathrm{C}$. Figure $1(\mathrm{~b})$ represents the log(Resistance) change of the $\mathrm{ZnO}$ QD sensor with time for various acetone concentrations $(0-50 \mathrm{ppm})$ at $430{ }^{\circ} \mathrm{C}$. The resistance of the $\mathrm{ZnO} Q \mathrm{QD}$ sensor for the acetone gas began to decrease at $32 \mathrm{~s}$, reaching the minimum value at $42 \mathrm{~s}$, and then recovered. The output signal changes of the
$\mathrm{ZnO}$ QD sensor at 32 and 42 s were 0.305 , $0.188,0.108,0.070,0.023,0.017$ and 0.001 for $50,25,10,5,1,0.5$ and $0.1 \mathrm{ppm}$ acetone, respectively. The change in the resistance due to the reaction with acetone decreased with decreasing acetone concentration.

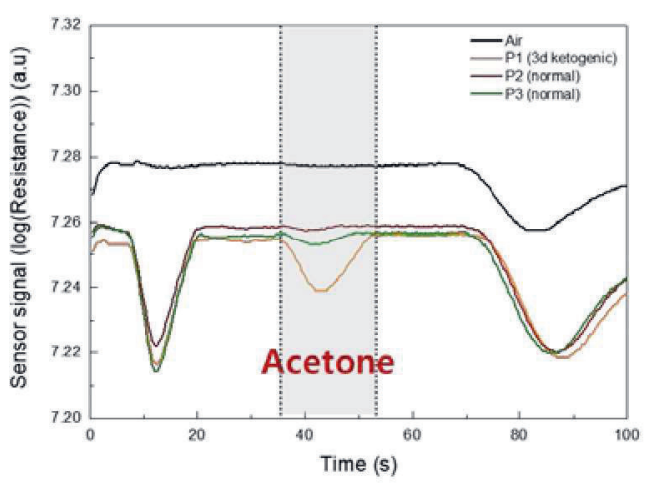

Fig. 2.Sensor signals for volunteers on ketogenic diet and normal diet for 3 days.

The breath acetone peak from the volunteer on the ketogenic diet was higher than the breath from the volunteers on a normal diet, $1.28 \mathrm{ppm}$ and $0.12 \mathrm{ppm}$, respectively. These values are similar to those mentioned in the previous report (before ketogenic diet: $0.17 \mathrm{ppm}$; after ketogenic diet for 3 days: $0.98 \mathrm{ppm}$ ) [7].

\section{Reference}

[1] L. Pauling, A. B. Robinson, Teranish.R, P. Cary, Quantitative Analysis of Urine Vapor and Breath by Gas-Liquid Partition Chromatography, Proc. Nat. Acad. Sci., 10, 2374 (1971); doi: 10.1073/pnas.68.10.2374

[2] W.Cao, Y. Duan, Breath Analysis: Potential for Clinical Diagnosis and Exposure Assessment, Clin. Chem., 52, 800 (2006); doi: 10.1373/clinchem.2005.063545

[3] A. Manolis, The diagnostic potential of breath analysis, Clin. Chem. 29 (1983) 5

[4] M. Statheropoulos, A. Agapiou, A. Georgiadou, Analysis of expired air of fasting male monks at mount athos, J. Chromatogr. B, 823, 274 (2006); doi: 10.1016/j.jchromb.2006.01.017

[5] G. Neri, A. Bonavita, G. Micali, N. Donato, Design and Development of a breath acetone MOS sensor for ketogenic diet control, IEEE Sens. Jour., $10, \quad 131 \quad$ (2010); doi: 10.1109/JSEN.2009.2035663

[6] M. Kupari, J. Lommi, M. Ventila, U. Karjalainen, Breath acetone in congestive heart failure, Am. J. Caridiol. 76, 1076 (1995); dor:: 10.1016/S00029149(99)80304-X

[7] P. Spanel, K. Dryahina, A. Rejskova, T.W.E. Chippendale, D. Smithm, Breath acetone concentration; biological variability and the influence of diet, Physiol. Meas., 32, N23 (2011); doi: 10.1088/0967-3334/32/8/N01 